\title{
Epigallocatechin-3-gallate exhibits anti-tumor effect by perturbing redox homeostasis, modulating the release of pro-inflammatory mediators and decreasing the invasiveness of glioblastoma cells
}

\author{
ANINDITA AGARWAL, VIVEK SHARMA, RICHA TEWARI, NITIN KOUL, \\ CHRISTY JOSEPH and ELLORA SEN
}

National Brain Research Centre, Manesar, Haryana 122 050, India

Received January 17, 2008; Accepted March 24, 2008

\begin{abstract}
Polyphenol epigallocatechin-3-gallate (EGCG) induced apoptosis in glioma cells by elevating oxidative stress through increased reactive oxygen species (ROS) generation. Signs of apoptosis included altered mitochondrial membrane potential and elevated expression of caspase- 3 and cytochrome $c$. The increase in ROS was concomitant with the decrease in expression of thioredoxin (TRX-1) and ceruloplasmin $(\mathrm{CP})$, mediators associated with protection against oxidative stress. EGCG downregulated the levels of pro-inflammatory cytokine interleukin (IL)-6 and chemokines IL-8, monocyte-chemoattractant protein (MCP)-1 and RANTES. EGCG also decreased the invasive potential of gliomas, possibly by affecting the urokinase plasminogen activator (UPA) and cytoskeletal architecture. Our study indicates that EGCG might serve as an effective therapeutic strategy against glioma as it not only promotes cell death through redox perturbation, but also downregulates the release of proinflammatory mediators while concomitantly decreasing the invasive potential of glioma cells.
\end{abstract}

\section{Introduction}

Polyphenol epigallocatechin-3-gallate (EGCG), which is present in green tea, has cancer chemopreventive effects in many animal tumor models (1). Epidemiological studies have moreover shown that the consumption of green tea can reduce the incidence of cancer (2). Glioblastoma multiforme (GBM) remains one of the most challenging solid cancers to treat due to its highly proliferative, angiogenic and invasive nature. Heightened oxidative stress appears to be characteristic of GBMs, with reactive oxygen species (ROS) increasing resistance to chemotherapy in gliomas (3). We and others have reported that human cancer cells with intrinsic oxidative stress

Correspondence to: Dr Ellora Sen, National Brain Research Centre, Near NSG Campus, Manesar, Gurgaon, Haryana 122 050, India E-mail: ellora@nbrc.res.in

Key words: epigallocatechin-3-gallate, homeostasis, glioblastoma are highly sensitive to ROS stress, and that promoting ROS generation can effectively bring about cell death $(4,5)$. As EGCG induces the apoptosis of malignant cells via the production of ROS $(6,7)$, we investigated whether this catechin could be used as a redox-modulating agent to induce apoptosis in glioma cells by elevating oxidative stress.

Inflammation is also a contributing factor in cancer development (8). Increased expression of pro-inflammatory mediators has been implicated as a possible prognostic indicator in GBM (9). EGCG not only inhibits the release of proinflammatory mediators in human prostate carcinoma cells (10), but is also known to prevent glioblastoma migration by downregulating matrix metalloproteinase (MMP) activities (11). We therefore investigated whether EGCG could affect the release of pro-inflammatory mediators from glioma cells, altering their invasive potential.

\section{Materials and methods}

Cell culture and treatment. Glioblastoma cell line U87MG, obtained from American Type Culture Collection (Manassas, VA), was cultured in DMEM supplemented with $10 \%$ FBS. On attaining semi-confluence, cells were switched to serumfree media (SFM). After $12 \mathrm{~h}$, cells were treated with 25,50 and $100 \mu \mathrm{M}$ EGCG (Sigma, St. Louis, MO) in SFM for $48 \mathrm{~h}$. Following treatment, the conditioned media from the cells were collected and stored at $-80^{\circ} \mathrm{C}$ for cytokine bead array, whereas cells were processed for immunoblot analysis, apoptosis bead array and ROS measurement. To determine the involvement of ROS in apoptosis, the ROS-specific inhibitor $\mathrm{N}$-acetylcysteine (NAC) was added to cells either alone or in combination with EGCG. All reagents were purchased from Sigma (St. Louis, MO) unless otherwise stated.

Measurement of ROS. Intracellular ROS generation in the control and EGCG-treated cells was monitored using $\mathrm{H}_{2} \mathrm{O}_{2}$ sensitive probe 5 (and 6)-chloromethyl-2',7'-dichlorodi-hydrofluorescein diacetate (CM- $\mathrm{H}_{2}$ DCFDA), as previously described (5). Briefly, cells were incubated with $\mathrm{H}_{2}$ DCFDA $(5 \mu \mathrm{M})$ for $30 \mathrm{~min}$ at $37^{\circ} \mathrm{C}$ and washed twice with PBS. Then, the fluorescent intensity of $5 \times 10^{5}$ cells was measured using a spectrofluorometer (excitation $500 \mathrm{~nm}$; emission $530 \mathrm{~nm}$ ). 
A

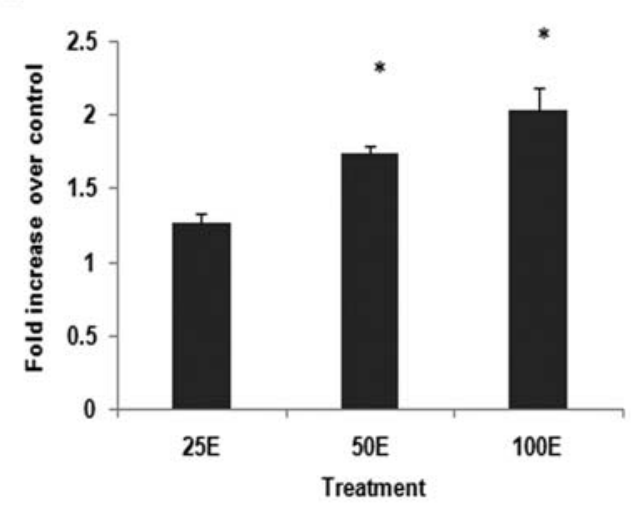

C

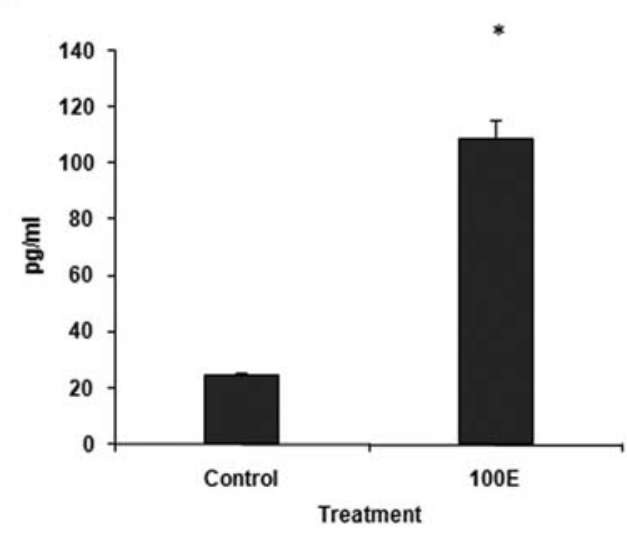

B

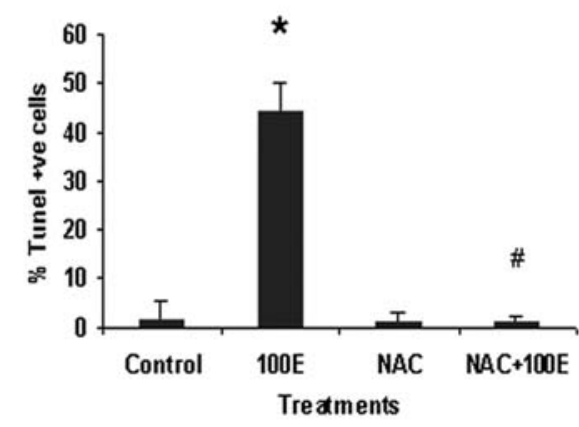

D

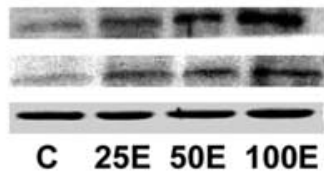

Cyt c

Bax

$\beta$-Tubulin

Figure 1. EGCG increases ROS production in glioma cells to induce apoptosis. (A) ROS levels were measured in cells treated with EGCG for 48 h using $\mathrm{H}_{2} \mathrm{O}_{2}-$ sensitive probe DCFDA. ROS levels following treatment with increasing concentrations of EGCG are indicated. (B) The ROS inhibitor NAC abolishes EGCGinduced cytotoxicity. Cell death was determined by TUNEL assay. The graph represents the percentage of TUNEL-positive cells treated with either $100 \mu \mathrm{M}$ EGCG, NAC, or a combination of both for $48 \mathrm{~h}$, as calculated from multiple fields. (C) Levels of active caspase-3 in the control and EGCG-treated U87MG cells, as observed by Apoptosis bead array. Values represent the means \pm SEM of 3 independent experiments. *Significant increase compared to the control; "significant decrease compared to EGCG-treated cells ( $\mathrm{p}<0.05)$. (D) Expression of cytochrome c and Bax in EGCG-treated cells as analyzed by Western blot analysis. A representative blot from three independent experiments with identical results is shown. Blots were reprobed for $\beta$-tubulin to establish equivalent loading. E, EGCG; C, control.

TUNEL assay. U87MG cells $\left(10^{4}\right)$ were treated with EGCG or $1 \mathrm{mM}$ NAC (a ROS-specific inhibitor) or a combination of both for $48 \mathrm{~h}$ in 8 -well chamber slides (Nunc, Denmark). Following treatment, apoptotic cells were identified using the In situ Cell Death Detection Kit, TMR red (Roche, Germany) as previously described (5). Cells fixed with $4 \%$ paraformaldehyde in PBS were blocked with $4 \%$ BSA containing $0.02 \%$ Triton X-100. Fixed cells were then incubated in the TUNEL mix (terminal deoxynucleotidyl transferase in storage buffer and TMR red-labeled nucleotide mixture in reaction buffer) for $1 \mathrm{~h}$ at room temperature. The slides were mounted with Vectashield mounting media containing DAPI (Vector Laboratories Inc., CA). TUNEL-positive cells (red) that colocalized with DAPI (blue) were counted from multiple fields.

Western blot analysis. Protein $(20 \mu \mathrm{g})$ isolated from untreated and EGCG-treated U87MG cells was electrophoresed on 6-10\% polyacrylamide gel. Western blotting was performed as previously described (5) using the following antibodies: ceruloplasmin (CP) (Dako, Carpinteria, CA), Bax, cytochrome $c$ and thioredoxin (TRX-1) (Lab Frontier, Seoul, Korea). Antibodies were purchased from Santa Cruz Biotechnology (Santa Cruz, CA) unless otherwise mentioned. Secondary anti- bodies were purchased from Vector Laboratories. Following the addition of chemiluminescence reagent (Amersham, Buckinghamshire, UK), blots were exposed to the Chemigenius Bioimaging System (Syngene, Cambridge, UK) for developing, and images were captured using GeneSnap software (Syngene). The blots were stripped and reprobed with anti-ß-tubulin to determine equivalent loading as previously described (5).

Determination of mitochondrial membrane potential. Mitochondrial membrane potential $\left(\Delta \Psi_{\mathrm{m}}\right)$ was measured using JC-1 probe as previously described (5). Control and EGCG-treated cells were labeled for 10 min with $10 \mu \mathrm{M} \mathrm{JC}-1$ at $37^{\circ} \mathrm{C}$, then cells were examined by fluorescence microscopy. JC-1 accumulates as J-aggregates (590 nm, red) in metabolically active mitochondria only, while the depolarization of mitochondrial membranes leads to JC-1 monomer formation (527 nm, green).

Cytometric bead array (CBA). The Human Inflammation Kit (BD Biosciences, NJ) was used to quantitatively measure cytokine/chemokine levels in the media obtained from untreated and EGCG-treated cells as previously described (5). The Human Apoptosis Kit (BD Biosciences) was used to 
A

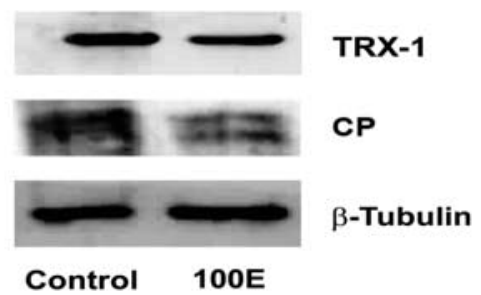

B

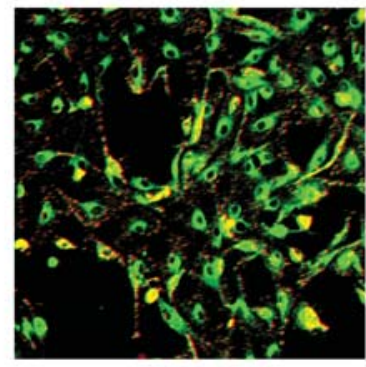

Control

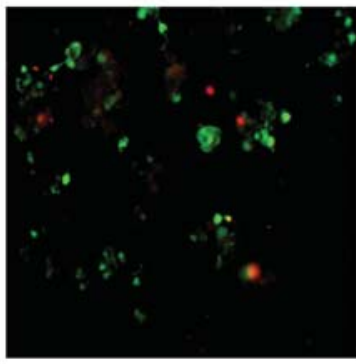

$100 E$
Figure 2. EGCG alters expression of the molecules associated with redox homeostasis and decreases the mitochondrial membrane potential of glioma cells. (A) Western blot showing the expression of TRX-1 and CP in the control and in cells treated with $100 \mu \mathrm{M}$ EGCG for $48 \mathrm{~h}$. Data are representative of results obtained from 3 independent experiments. Blots were reprobed for B-tubulin to establish equivalent loading. (B) Fluorescence micrographs of cells stained with $\mathrm{JC}-1$ show the decrease in mitochondrial membrane potential in cells treated with $100 \mu \mathrm{M}$ EGCG. Micrographs show that JC-1 accumulates within active mitochondria and exhibits orange fluorescence. E, EGCG; TRX-1, thioredoxin; CP, ceruloplasmin.

quantitatively measure caspase- 3 levels in the protein lysates of untreated and EGCG-treated cells. The assay was performed according to the manufacturer's instructions and analyzed on FACSCalibur (Becton Dickinson) using CBA software as previously described (5).

Matrigel invasion assay. In vitro invasion of U87MG cells grown in the absence or presence of EGCG for $48 \mathrm{~h}$ was measured by the invasion of cells through Matrigel-coated (Collaborative Research Inc., Boston, MA) transwell inserts (Costar, Cambridge, MA) as previously described (12). Briefly, 2x10 cells were added to transwell inserts with an $8-\mu \mathrm{m}$ pore size coated with a final concentration of $0.7 \mathrm{mg} / \mathrm{ml}$ Matrigel. Following treatment, cells that passed through the filter on the bottom side of the membrane were fixed, stained with crystal violet, photographed and quantitated as described (12).

Urokinase plasminogen activator assay. The assay was performed according to the manufacturer's instructions (Chemicon). Cell lysates from treated and untreated cells and the positive control (urokinase plasminogen activator (uPA)containing sample) were added to each well of a 96-well plate, and the volume was adjusted with deionized water. Following the addition of assay buffer and chromogenic substrate, the samples were incubated for $24 \mathrm{~h}$ at $37^{\circ} \mathrm{C}$, following which the absorbance was read at $405 \mathrm{~nm}$. Optical density values obtained were compared with known standards to obtain relative activities.
Visualization of actin cytoskeleton. Cells $\left(10^{4}\right)$ were treated with EGCG in 8-well chamber slides. After $48 \mathrm{~h}$, cells were washed with PBS and fixed with $4 \%$ formaldehyde for $10 \mathrm{~min}$ at $37^{\circ} \mathrm{C}$. Fixed cells were then incubated in PBS containing $1 \%$ BSA for $30 \mathrm{~min}$, then in staining solution $(6.25 \mu 1$ rhodaminelabeled phalloidin, $2.5 \mathrm{mg}$ BSA in $250 \mu \mathrm{l}$ PBS) for $20 \mathrm{~min}$ at room temperature. Cells were washed with PBS, mounted, and immunofluorescence was recorded using a fluorescence microscope.

Statistical analysis. All comparisons between groups were performed using the two-tailed paired Student's t-test. Values of $\mathrm{p}<0.05$ indicated significant differences.

\section{Results}

EGCG enhances ROS production in glioblastoma cells to induce apoptosis. While treatment of U87MG cells with $25 \mu \mathrm{M}$ EGCG had no effect on ROS production, a significant 1.7- and 2 -fold $(\mathrm{p}<0.05)$ increase in ROS production was observed in cells treated with 50 and $100 \mu \mathrm{M}$ EGCG respectively, as compared to the control cells (Fig. 1A). To determine whether increased ROS production induced apoptosis in glioma cells, TUNEL staining was performed on cells treated with the ROS inhibitor NAC in the presence and absence of $100 \mu \mathrm{M}$ EGCG (this dose was selected as maximal ROS production was observed at this concentration). No significant difference in the percentage of apoptotic cells was observed between untreated and NAC-treated cell (Fig. 1B). However, the 22-fold increase in apoptotic cells observed when U87MG cells were treated with $100 \mu \mathrm{M}$ EGCG was significantly reduced to that of the control level when EGCG treatment was supplemented with NAC (Fig. 1B). Thus, the cytotoxicity of EGCG was abolished in the presence of ROS inhibitor.

Since significant apoptosis (42\%, Fig. 1B) was observed in U87MG cells treated with $100 \mu \mathrm{M}$ of EGCG, we performed FACS analysis to detect the levels of pro-apoptotic molecule caspase-3 in EGCG-treated cells. A significant 5-fold increase in the expression of caspase-3 was observed in EGCG-treated cells as compared to the control cells (Fig. 1C). Bax, a proapoptotic protein, promotes the release of cytochrome $c$ from the mitochondria, in turn activating caspase-3, one of the key executioners of apoptosis (13). Since increased caspase-3 levels were observed in EGCG-treated cells, we determined Bax and cytochrome $c$ levels in EGCG-treated cells by Western blot analysis. A significant increase in both Bax and cytochrome $c$ expression was observed in cells treated with 50 and $100 \mu \mathrm{M}$ EGCG (Fig. 1D).

EGCG decreases expression of the molecules associated with the maintenance of redox homeostasis. Since a major cellular defense against ROS is provided by the antioxidant TRX-1, we evaluated the status of redox protein TRX-1 in EGCG-treated glioma. Additionally, the level of $\mathrm{CP}$, an antioxidant that protects astrocytes from sustaining damage after injury, was investigated. Treatment of glioma cells with $100 \mu \mathrm{M}$ of EGCG dramatically decreased the expression of both TRX-1 and CP as compared to the untreated control cells (Fig. 2A).

EGCG decreases mitochondrial membrane potential in human glioma cells. As ROS generation is inversely correlated with 


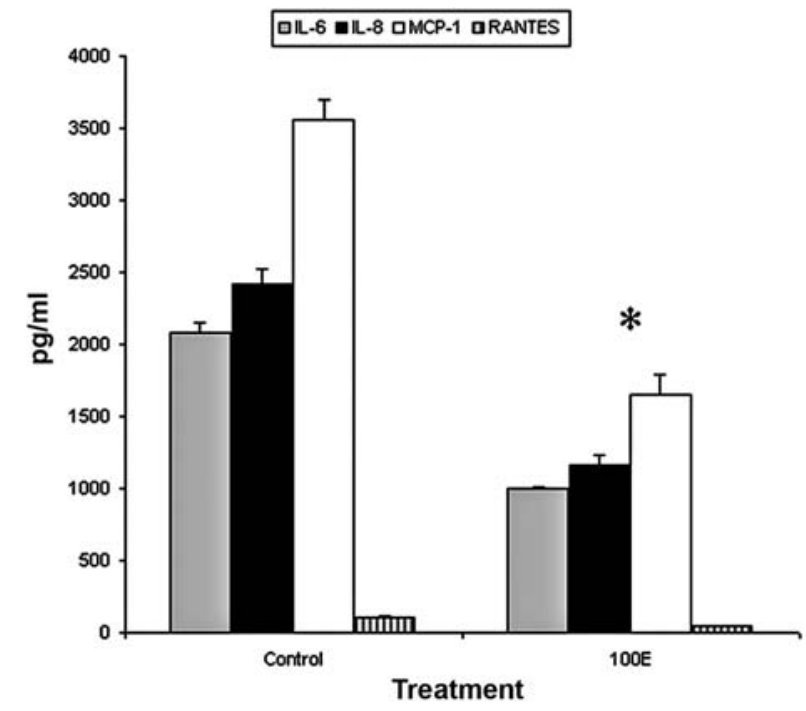

Figure 3. EGCG modulates the release of cytokines/chemokines from glioblastoma. Expression of the cytokine IL-6 and chemokines IL-8, MCP-1 and RANTES in untreated and EGCG-treated U87MG cells, as observed by CBA. Values represent the means \pm SEM of 3 individual experiments. ${ }^{*}$ Significant decrease compared to the control $(\mathrm{p}<0.05)$. E, EGCG.

mitochondrial membrane potential $\left(\Delta \Psi_{\mathrm{m}}\right)(14)$, and since increased release of cytochrome $c$ in EGCG-treated cells indicates mitochondrial involvement, we investigated mitochondrial changes in terms of alterations in $\Delta \Psi_{\mathrm{m}}$. Intact mito- chondrial function, as indicated by the accumulation of JC-1 orange fluorescence, was observed in untreated cells (Fig. 2B). The absence of detectable JC-1 orange fluorescence observed in cells treated with $100 \mu \mathrm{M}$ EGCG indicated diminished $\Delta \Psi_{\mathrm{m}}$ (Fig. 2B).

EGCG decreases the release of cytokines/chemokines from glioma cells. Pro-inflammatory cytokine interleukin (IL)-6 plays a role in the malignant progression of astrocytoma (15), and chemokines such as IL-8, monocyte-chemoattractant protein (MCP-1) and RANTES are crucial regulators of cancer cell invasion (16). Since EGCG inhibits pro-inflammatory mediator release in human prostate carcinoma cells (10), we performed CBA to investigate the profile of cytokines/chemokines in EGCG-treated cells. A significant 2 -fold decrease in IL-6, IL-8, MCP-1 and RANTES levels was observed in EGCGtreated cells as compared to the control cells (Fig. 3).

EGCG decreases the invasiveness of glioma cells. EGCG prevents GBM migration by downregulating MMP activity (11). Since EGCG treatment decreases levels of IL-8, MCP-1 and RANTES, which are associated with cancer cell invasion (16), Matrigel invasion assay was performed to determine the effect of EGCG on glioma cell invasiveness. As a concentration of $100 \mu \mathrm{M}$ EGCG was most effective in inducing ROS-mediated apoptosis, this dose was chosen for the Matrigel invasion assay. A $60 \%$ decrease in the invasive potential of glioma was observed upon treatment with $100 \mu \mathrm{M}$ of EGCG (Fig. 4A).
A

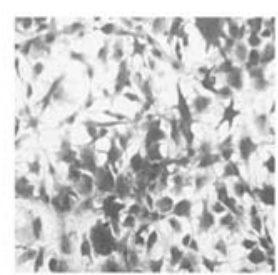

Control

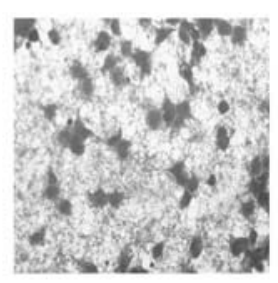

$100 \mathrm{E}$
B

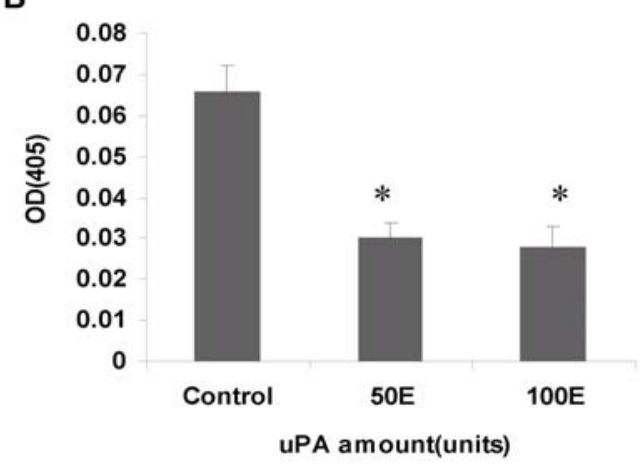

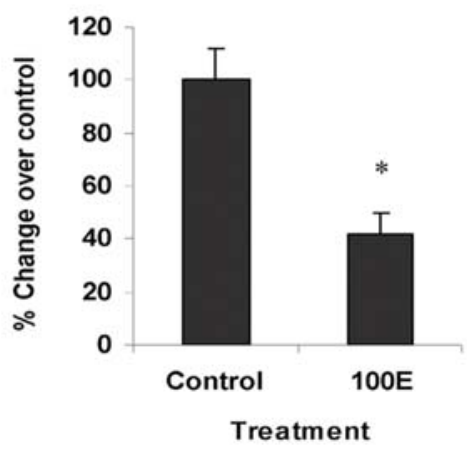

C
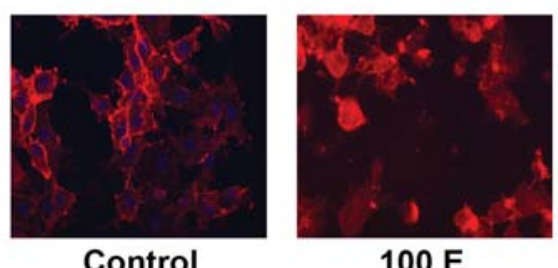

Figure 4. EGCG decreases the invasiveness of glioma cells. (A) EGCG decreases the invasiveness of glioma cells as determined by Matrigel invasion assay. Control and EGCG-treated cells were allowed to pass through a Matrigel chamber; the cells that invaded the bottom side of the membrane were fixed, stained with crystal violet and photographed. The graph represents the percentage of glioma cells treated with EGCG that invaded the Matrigel. (B) EGCG decreased the $\mathrm{uPa}$ activity of glioma cells. The uPa activity of cell lysates obtained from EGCG-treated U87MG cells was measured, and the absorbance was read at 405 nm. The optical density values were compared with known standards to obtain relative activities. The graph represents optical density as a measure of uPa activity in the control and EGCG-treated cells. Values represent the means \pm SEM of 3 independent experiments. * Significant decrease compared to the control (p<0.05). (C) EGCG alters the cytoskeletal architecture of GBM by affecting F-actin condensation as visualized after staining cells with rhodamine-labeled phalloidin. E, EGCG. 
The binding of urokinase plasminogen activator (uPA) to its receptor (UPAR) initiates a proteolytic cascade facilitating the activation of MMP, which in turn degrades the extracellular matrix (12). As EGCG suppressed uPA expression in human fibrosarcoma (17), and since EGCG decreased the invasiveness of U87MG cells (Fig. 4A), we determined uPA activity in glioma cells treated with EGCG (Fig. 4B). A 40-50\% decrease in $\mathrm{uPa}$ activity was observed in glioma cells upon treatment with 50 and $100 \mu \mathrm{M}$ of EGCG (Fig. 4B).

Actin-binding proteins influence microfilament dynamics to facilitate the invasiveness of malignant cells (18). Since EGCG downregulated the invasive potential of U87MG cells, we stained glioma cells with phalloidin (which binds specifically to F-actin) to investigate the distribution of F-actin in the presence and absence of EGCG (Fig. 4C). A decrease in the actin network was observed in EGCG-treated cells, as evidenced by decreased staining with phalloidin as compared to the control. The disassembly of the network indicated that EGCG affects cytoskeletal architecture to decrease the invasiveness of glioblastoma cells.

\section{Discussion}

Despite recent advances in understanding the molecular pathways involved in GBM aggressiveness, the prognosis for this most malignant of brain tumors continues to be dismal. As EGCG suppresses the growth of a number of human malignancies, we investigated the molecular mechanism of the action of EGCG on the human GBM cell line U87MG. It is known that intrinsic ROS stress renders cancer cells highly vulnerable to further oxidative insults by exogenous agents $(5,6,19)$. Our study indicated that EGCG induces apoptosis in glioma cells through increased oxidative stress. Increased apoptosis in glioma with increased ROS levels and downregulated TRX-1 are consistent with previous reports that the inhibition of TRX enhances the sensitivity of tumor cells to ROS-generating anticancer agents $(5,20)$. An increase in ROS with a concomitant decrease in antioxidant TRX-1 and ferroxidase CP possibly enhances oxidative stress in EGCG-treated glioma cells by decreasing their ability to detoxify excess free radicals.

ECGC-mediated depolarization of the mitochondrial membrane likely facilitated the release of apoptogenic protein cytochrome $c$, as previously observed in pancreatic cancer cells (7). We speculate that EGCG-mediated downregulation of IL-6, IL-8, RANTES and MCP-1 possibly prevents the establishment of the feed forward cycle of inflammation that promotes disease progression. The ability of EGCG to decrease the invasive potential of glioma cells was concurrent with a decrease in the $\mathrm{uPa}$ activity associated with invasiveness.

Our study demonstrates that the ability of EGCG to (i) induce oxidative stress in glioma cells by disrupting the redox homeostasis and (ii) downregulate the expression of pro-inflammatory mediators may be an attractive anti-tumor strategy, given the role of inflammation and oxidative stress in GBM progression

\section{Acknowledgements}

This work was supported by a research grant from the Defense Research and Development Organization, The Government of India, to E.S.

\section{References}

1. Yang CS and Wang ZY: Tea and cancer. J Natl Cancer Inst 85: 1038-1049, 1993.

2. Ahmad N, Feyes DK, Nieminen AL, Agarwal R and Mukhtar H: Green tea constituent epigallocatechin-3-gallate and induction of apoptosis and cell cycle arrest in human carcinoma cells. J Natl Cancer Inst 89: 1881-1886, 1997.

3. Silber JR, Bobola MS, Blank A, Schoeler KD, Haroldson PD, Huynh MB and Kolstoe DD: The apurinic/apyrimidinic endonuclease activity of Ape1/Ref-1 contributes to human glioma cell resistance to alkylating agents and is elevated by oxidative stress. Clin Cancer Res 8: 3008-3018, 2002.

4. Schumacker PT: Reactive oxygen species in cancer cells: live by the sword, die by the sword. Cancer Cell 10: 175-176, 2006.

5. Sharma V, Joseph C, Ghosh S, Agarwal A, Mishra MK and Sen E: Kaempferol induces apoptosis in glioblastoma cells through oxidative stress. Mol Cancer Ther 6: 2544-2553, 2007.

6. Nakazato T, Ito K, Ikeda Y and Kizaki M: Green tea component, catechin, induces apoptosis of human malignant B cells via production of reactive oxygen species. Clin Cancer Res 11: 6040-6049, 2005

7. Qanungo S, Das M, Haldar S and Basu A: Epigallocatechin3 -gallate induces mitochondrial membrane depolarization and caspase-dependent apoptosis in pancreatic cancer cells. Carcinogenesis 26: 958-967, 2005

8. Coussens LM and Werb Z: Inflammation and cancer. Nature 420: 860-867, 2002.

9. Chang CY, Li MC, Liao SL, Huang YL, Shen CC and Pan HC: Prognostic and clinical implication of IL-6 expression in glioblastoma multiforme. J Clin Neurosci 12: 930-933, 2005.

10. Adhami VM, Malik A, Zaman N, Sarfaraz S, Siddiqui IA, Syed DN, Afaq F, Pasha FS, Saleem M and Mukhtar H: Combined inhibitory effects of green tea polyphenols and selective cyclooxygenase- 2 inhibitors on the growth of human prostate cancer cells both in vitro and in vivo. Clin Cancer Res 13: 1611-1619, 2007.

11. Annabi B, Lachambre MP, Bousquet-Gagnon N, Page M, Gingras D and Beliveau R: Green tea polyphenol (-)-epigallocatechin 3-gallate inhibits MMP-2 secretion and MT1-MMPdriven migration in glioblastoma cells. Biochim Biophys Acta 1542: 209-220, 2002 .

12. Lakka SS, Gondi CS, Yanamandra N, Dinh DH, Olivero WC, Gujrati M and Rao JS: Synergistic down-regulation of urokinase plasminogen activator receptor and matrix metalloproteinase-9 in SNB19 glioblastoma cells efficiently inhibits glioma cell invasion, angiogenesis, and tumor growth. Cancer Res 63: 2454-2461, 2003.

13. Kluck RM, Bossy-Wetzel E, Green DR and Newmeyer DD: The release of cytochrome $\mathrm{c}$ from mitochondria: a primary site for Bcl-2 regulation of apoptosis. Science 275: 1132-1136, 1997.

14. Campian JL, Qian M, Gao X and Eaton JW: Oxygen tolerance and coupling of mitochondrial electron transport. J Biol Chem 279: 46580-46587, 2004

15. Loeffler S, Fayard B, Weis J and Weissenberger J: Interleukin-6 induces transcriptional activation of vascular endothelial growth factor (VEGF) in astrocytes in vivo and regulates VEGF promoter activity in glioblastoma cells via direct interaction between STAT3 and Sp1. Int J Cancer 115: 202-213, 2005.

16. Dinarello CA: The paradox of pro-inflammatory cytokines in cancer. Cancer Metastasis Rev 25: 307-313, 2006.

17. Kim MH, Jung MA, Hwang YS, Jeong M, Kim SM, Ahn SJ, Shin BA, Ahn BW and Jung YD: Regulation of urokinase plasminogen activator by epigallocatechin-3-gallate in human fibrosarcoma cells. Eur J Pharmacol 487: 1-6, 2004.

18. Verschueren H, van der Taelen I, Dewit J, De Braekeleer J and De Baetselier P: Metastatic competence of BW5147 T-lymphoma cell lines is correlated with in vitro invasiveness, motility and F-actin content. J Leukoc Biol 55: 552-556, 1994.

19. Trachootham D, Zhou Y, Zhang H, Demizu Y, Chen Z, Pelicano H, Chiao PJ, Achanta G, Ralph B. Arlinghaus RB, Liu J and Huang P: Selective killing of oncogenically transformed cells through a ROS-mediated mechanism by beta-phenylethyl isothiocyanate. Cancer Cell 10: 241-252, 2006.

20. Ungerstedt JS, Sowa Y, Xu WS, Shao Y, Dokmanovic M, Perez G, Ngo L, Holmgren A, Jiang X and Marks PA: Role of thioredoxin in the response of normal and transformed cells to histone deacetylase inhibitors. Proc Natl Acad Sci USA 102: 673-678, 2005. 